\title{
Neutron Diffraction Texture Analysis using a $2 \theta$-Position Sensitive Detector
}

\author{
H. J. BUNGE, † H. R. WENK $\ddagger$ and J. PANNETIER
}

\begin{abstract}
†Institut für Metallkunde und Metallphysik, Technische Universität Clausthal, Germany

$\ddagger$ Department of Geology and Geophysics, University of California, Berkeley, USA $\S$ Institut Max von Laue-Paul Langevin, Grenoble, France
\end{abstract}

(Dedicated to Prof. Dr. Dr. h.c. G. Wasserman on the occasion of his 80th birthday)

(Received January 5, 1982; in final form May 5, 1982)

\begin{abstract}
A method for pole figure measurements in textured samples using neutron diffraction and a position sensitive detector is described. The position sensitive detector allows one to record the whole 29-spectrum of one sample position simultaneously, i.e. in reasonable time. The availability of the complete $2 \vartheta$ spectrum allows separation of overlapping peaks by a deconvolution process. A second independent method of peak separation is based on the crystallographic relations between peaks of various indices $(\mathrm{hkl})$. The method allows extraction of the maximum possible information about the texture out of a polycrystal diffraction spectrum. It is thus especially suited to texture studies in materials having complex diffraction spectra with overlapping peaks, i.e. materials with low crystal symmetry and large lattice parameters as they are frequently encountered in geology.
\end{abstract}

\section{INTRODUCTION}

The texture of a polycrystalline material is the orientation distribution of its crystallites with respect to a sample fixed reference system e.g. rolling plane and rolling direction in metallic materials or mesoscopic and macroseopic fabric co-ordinates in geological samples. Textures of materials are of two-fold interest. First, the texture determines the orientation dependent mean values of anisotropic properties in polycrystalline samples e.g. the strength of a material in various sample directions or the Youngs modulus and hence the sound velocity in rocks. Secondly, the texture depends on the history of the 
material such as plastic deformation, phase transformations and recrystallization. The texture can thus be used as an indicator for the history of a material. This latter aspect is especially relevant in geology where it provides information about processes which have taken place millions of years ago.

The most commonly used experimental data in texture analysis, besides orientation measurements of individual crystallites e.g. on the universal stage microscope, are pole figures. These are the orientation distributions of specific crystal directions, the normals to reflecting lattice planes (Wassermann and Grewen, 1962). Since an individual pole figure in general does not allow distinction between crystal orientations obtained by a rotation about the normal to the reflecting lattice plane, it needs a certain set of pole figures (the number of which increases with decreasing crystal symmetry) in order to determine the orientation distribution function of the crystallites.

In the earlier texture studies pole figures have been interpreted qualitatively in terms of crystal orientations by looking at peak intensities in the pole figures belonging to different directions $(h k l)$ of one and the same crystal orientation. Pole figures were thus interpreted qualitatively in terms of so called "ideal orientations" (Wassermann and Grewen, 1962). Recently, methods have been developed to calculate quantitatively the orientation distribution function from several pole distribution functions (pole figure inversion). These methods are no longer restricted to a few ideal orientations, rather they consider the texture as a continuous orientation distribution function (for a short summary see Bunge, 1981). The experimental starting point of these calculations are quantitatively determined pole distribution functions (pole figures) the number of which must be the larger the lower the crystal symmetry.

Pole figures have usually been measured by $\mathrm{X}$-ray diffraction. They can, however, also be measured by neutron diffraction using very similar methods to those used in X-ray diffraction. The main advantages of neutron diffraction result from the lower absorption of neutrons with respect to X-rays in most materials. This allows much bigger sample volumes to be transmitted, and this in turn increases the crystallite statistics and thus provides much more accurate and statistically more relevant results compared to $\mathrm{X}$-ray measurements.

The pole figure inversion method has first been applied to metals having high symmetries and small unit cell parameters. In this case the needed pole figures are well separated with respect to the Bragg angle $\theta$. The usual method used to measure pole figures is to fix the detector at the appropriate 20 position, the width of the receiving slit $\Delta 2 \theta$ being large enough to cover the whole diffraction profile of the peak $(h k l)$ being measured. This method breaks down with increasing number of reflection peaks i.e. with increasing lattice parameters and with decreasing symmetry as is usually the case in 
geological materials. Overlap of diffraction peaks then becomes more and more frequent. In this case the integrated intensity method is no longer applicable. Continuous $\theta$-scans are then needed which allow the separation of overlapping diffraction peaks by a peak profile analysis (see later section). This method needs, however, a much larger number of experimental data. Whereas one intensity value is sufficient in order to obtain the integrated intensity of the $(h k l)$-reflection, at least ten times as many points are necessary in order to carry out a profile analysis. This effect still adds to the increased number of pole figures $(h k l)$ needed in these cases. (In the present example pole figure measurements at about $200 \theta$-values have been used compared to 3 or 4 which are usual in texture studies of cubic metals.) With the conventional X-ray or neutron diffraction methods, using one detector only, this would unduly increase the necessary measuring time and this method would thus be prohibitive. If, however, a position sensitive detector is used a complete $\theta$-spectrum can be measured in sufficiently small steps $\Delta \theta$ at the same time, thus reducing the required measuring time to practicable values.

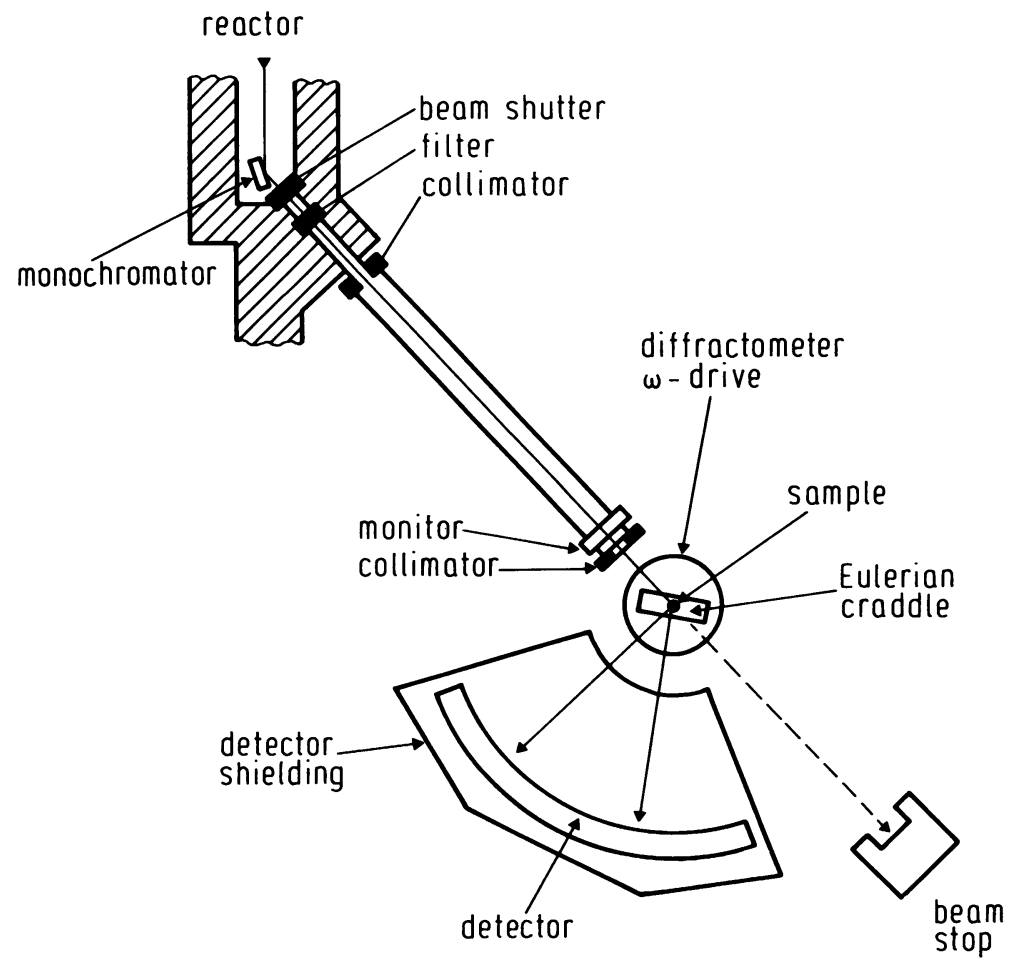

FIGURE 1 Schematic view of the two-axis powder diffractometer DIB with Eulerian cradle. (ILL Grenoble) adapted from ILL manual. 
The use of a position sensitive detector leads, however, to different geometrical conditions to those used in conventional pole figure measurements. It is thus necessary to use a modified pole figure scanning schedule which will be described in the following.

\section{THE NEUTRON DIFFRACTOMETER}

The diffractometer used for the measurements was the two-axis diffractometer DIB (Allemand et al., 1975) of the Institut Laue-Langevin at Grenoble, a schematic drawing of which is shown in Figure 1. A flux of monochromatic thermal neutrons is provided by a neutron guide and a pyrolytic graphite (0002) monochromator which selects a wavelength of $\lambda=2.522 \AA$, the higher order harmonics being suppressed by a graphite filter (Loopstra, 1966). The average flux at the sample is about $1.5 \cdot 10^{6} \mathrm{n} \mathrm{cm}^{-2} \mathrm{~s}^{-1}$. Temporal fluctuations of the neutron source are eliminated by the use of a monitor counter. The diffracted intensity is collected in a position sensitive $\mathrm{BF}_{3}$ multidetector with 400 cells covering $80^{\circ}$ in $2 \theta$ with 5 cells per degree $2 \theta$. The height of the cells is $100 \mathrm{~mm}$ which defines the angular resolving power. The sample to detector distance is $1.5225 \mathrm{~m}$. The counter can be moved about the sample so that an angular range $2^{\circ} \approx 2 \theta \approx 160^{\circ}$ can be covered. The efficiency of the detector is about $45 \%$ at $2.5 \AA$. The dispersion of cell efficiencies is about $5 \%$; therefore the 400 cells were calibrated before the measurement by using the diffusion pattern of a vanadium rod (Convert, 1975). The counting stability of each cell is estimated to be better than $0.3 \%$ over several days. With a measuring time of 9 minutes for each angle setting, an accuracy of the intensity measurement of about $0.5 \%$ was possible. The resolution of the instrument depends both on beam and monochromator characteristics (Gaglioti et al., 1958) and sample size. With a $10 \mathrm{~mm}$ diameter sample, the full width at half maximum (FWHM) is minimum at low angle (FWHM $\sim 0.40^{\circ}(2 \theta)$ at $2 \theta=30^{\circ}$ ) but increases rapidly with increasing Bragg angle (FWHM $\sim 0.80$ at $2 \theta=80^{\circ}$ ).

\section{THE EULERIAN CRADLE}

A Eulerian cradle which can be rotated around the $\omega$-axis is mounted on the diffractometer (Figure 2). The cradle allows the sample to be rotated about two angles $\chi$ and $\varphi$ (Figure 2). The cradle has a shadow range of $38^{\circ}$ at the side of the incident beam and of $27^{\circ}$ at the side of the reflected beam (the two sides can, however, be exchanged by a $180^{\circ}$-rotation (Figure 3 ).

When measuring pole figures one by one with peak maximum geometry aparatus, the plane of the $\chi$-rotation is chosen such that it contains the diffraction vector i.e. the bisectrix between incident and reflected beam. In 
the multicounter mode this is only possible for one specific reflection at the angle $2 \vartheta_{m}$ (Figure 4) which may be chosen as the middle of the $2 \vartheta$-range to be used.

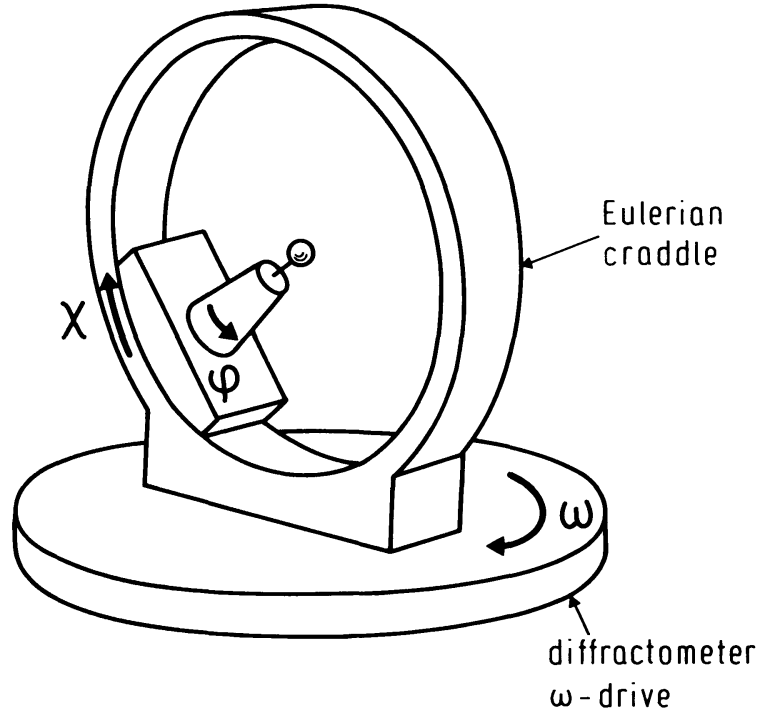

FIGURE 2 Schematic view of the Eulerian cradle mounted on the $\omega$-axis of the diffractometer.

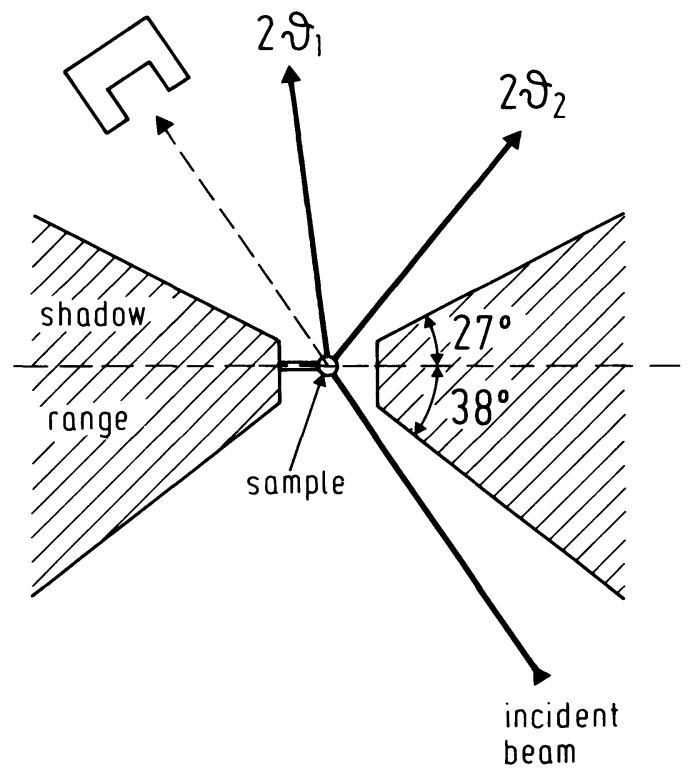

FIGURE 3 Shadow ranges of the Eulerian cradle. 


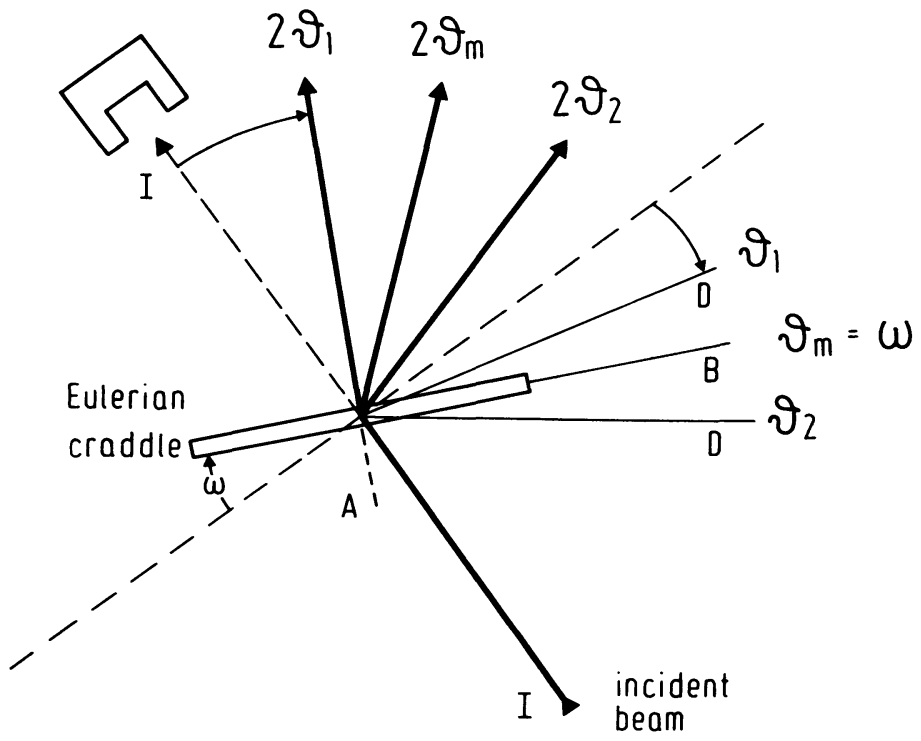

FIGURE 4 Positioning of the Eulerian cradle in the middle of the 9-range to be used. The letters A,B,D,I correspond to points in Figures 6 and 7.

\section{THE SCANNING OF THE POLE FIGURES}

The plane of the $\chi$-motion in the sample position $\varphi=0$ is chosen as the reference plane of the pole figure (Figure 5a). The position of the diffraction vector in the pole figure is described by the angular coordinates $\alpha$ and $\beta$ as is shown in Figure $5 \mathrm{~b}$. We consider the pole figure corresponding to a reflection at $2 \vartheta_{i}$. In the position of the sample $\chi=0, \varphi=0$ the diffraction vector is at the point $\mathrm{D}$ with the coordinates $\alpha=\vartheta_{i}-\omega, \beta=0$ as is shown in Figure 6 (compare Figure 4). Rotation of the sample through the angle $\chi$ brings the diffraction vector into the point $C$ with the coordinates $\alpha, \beta_{o}$ which can be obtained from the spherical triangle $A B C$ by application of the sine and cosine law of spherical trigonometry. This leads to the relations

$$
\begin{aligned}
& \cos \alpha=\cos \left(\vartheta_{i}-\omega\right) \cdot \cos \chi \\
& \sin \beta_{o}=\cos \left(\vartheta_{i}-\omega\right) \cdot \frac{\sin \chi}{\sin \alpha}
\end{aligned}
$$

Since $\alpha$ may vary in the range $0 \leqslant \alpha \leqslant 180^{\circ}$ and $\beta_{o}$ in the range $-90^{\circ} \leqslant \beta_{o} \leqslant+90^{\circ}$ these angles are completely determined by Eq. (1) and (2). A further rotation of the sample through $\varphi$ brings the diffraction vector into the point $\mathrm{E}$ with the same angle $\alpha$ and with 


$$
\beta=\beta_{o}+\varphi
$$

In the case $\vartheta_{i}=\omega$ the Eqs. (1)-(3) take on the simple form

$$
\alpha=\chi, \beta=90^{\circ}+\varphi
$$

which corresponds to the conventional pole figure measurement.

In the case $\vartheta_{i}<\omega$ the small-circle passing through point $\mathrm{D}$ is at the lower hemisphere of the pole figure. In this case $\beta_{o}$ is to be replaced by $180^{\circ}-\beta_{o}$. The Eqs. (1) $-(3)$ are thus changed into Eqs. $\left(1^{\prime}\right)-\left(3^{\prime}\right)$.

$$
\begin{gathered}
\cos \alpha=\cos \left(\omega-\vartheta_{i}\right) \cdot \cos \chi \\
\sin \beta_{o}=\cos \left(\omega-\vartheta_{i}\right) \frac{\sin \chi}{\sin \alpha} \\
\beta=\beta_{o}+\varphi
\end{gathered}
$$

where $\beta_{o}$ is in the range $90 \leqslant \beta_{o} \leqslant 270^{\circ}$.

The two cases $\vartheta_{i}>\omega$ and $\vartheta_{i}>\omega$ are better illustrated in Figure 7 which is rotated through $90^{\circ}$ with respect to Figure 6.

Varying the angles $\chi$ and $\varphi$ in the complete range $0 \leqslant \chi \leqslant 360^{\circ}$ and $0 \leqslant \varphi \leqslant$ $360^{\circ}$ does not yield the complete pole figure. In the general case, only the hatched area in Figure 8 is obtained. On the other hand the same point $\alpha \beta$

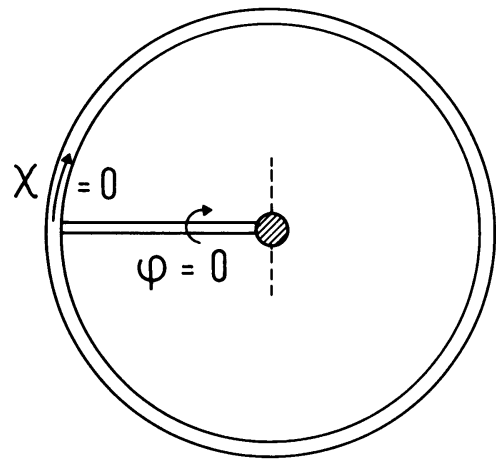

0

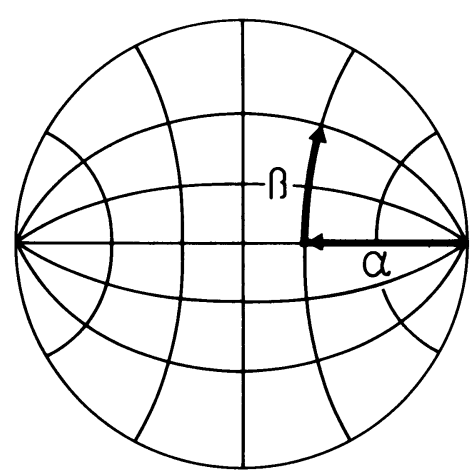

b

FIGURE 5 Definition of the projection plane of the pole figure.

(a) The projection plane (fixed to the sample) is the plane of the $\chi$-motion in the sample position $\chi=0, \varphi=0$. A rotation of the sample through $90^{\circ}$ about the dashed axis leads to the second setting (compare Figure 11).

(b) The angles $\alpha$ and $\beta$ are used to describe the position of the reflection vector in the sample orientation $\chi \varphi$. 


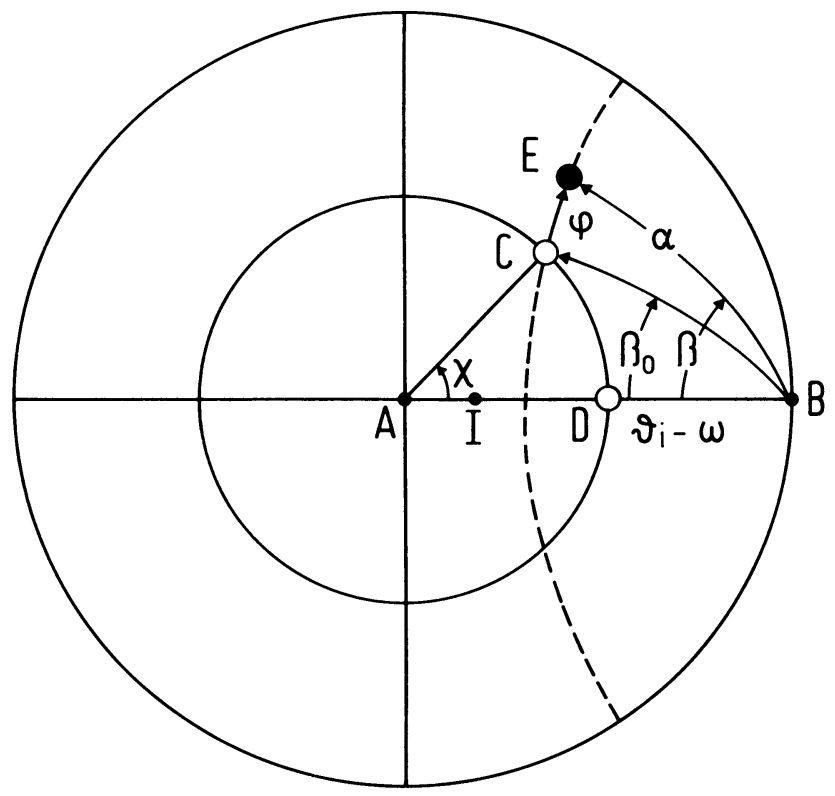

FIGURE 6 Relation between the pole figure angles $\alpha \beta$ and the sample angles $\chi \varphi$ corresponding to the Bragg angle $\vartheta_{i}$ and the position $\omega$ of the Eulerian cradle. For the points A,B,D,I compare Figure 4.

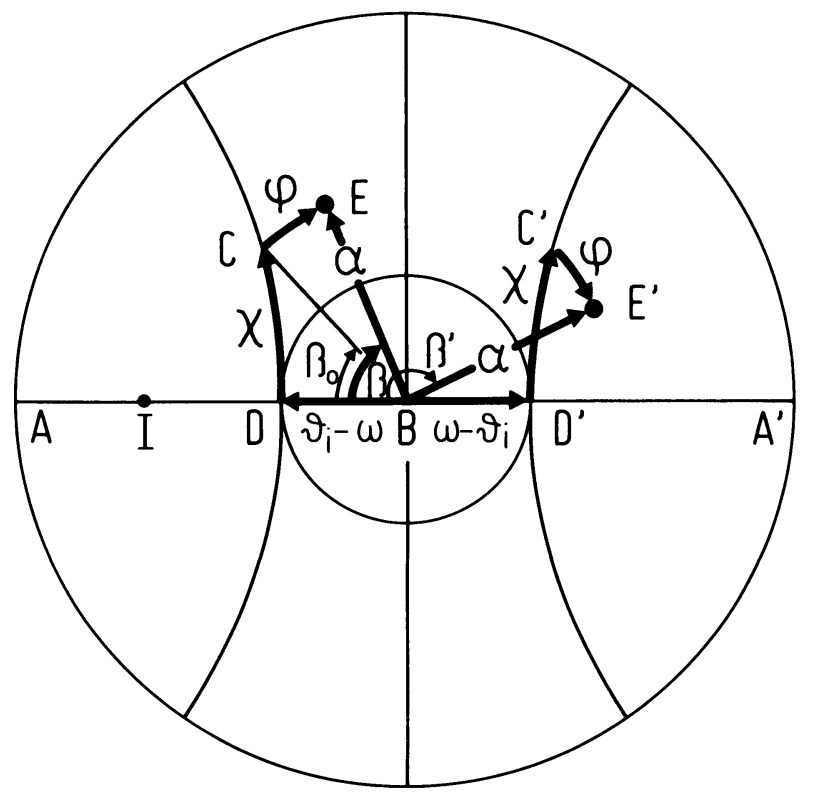

FIGURE 7 Position of the diffraction vector $\mathrm{E}\left(\mathrm{E}^{\prime}\right)$ in the two cases $\vartheta_{i}>\omega$ and $\vartheta_{i}<\omega$. The plane of projection is rotated through $90^{\circ}$ with respect to Figure 6. 


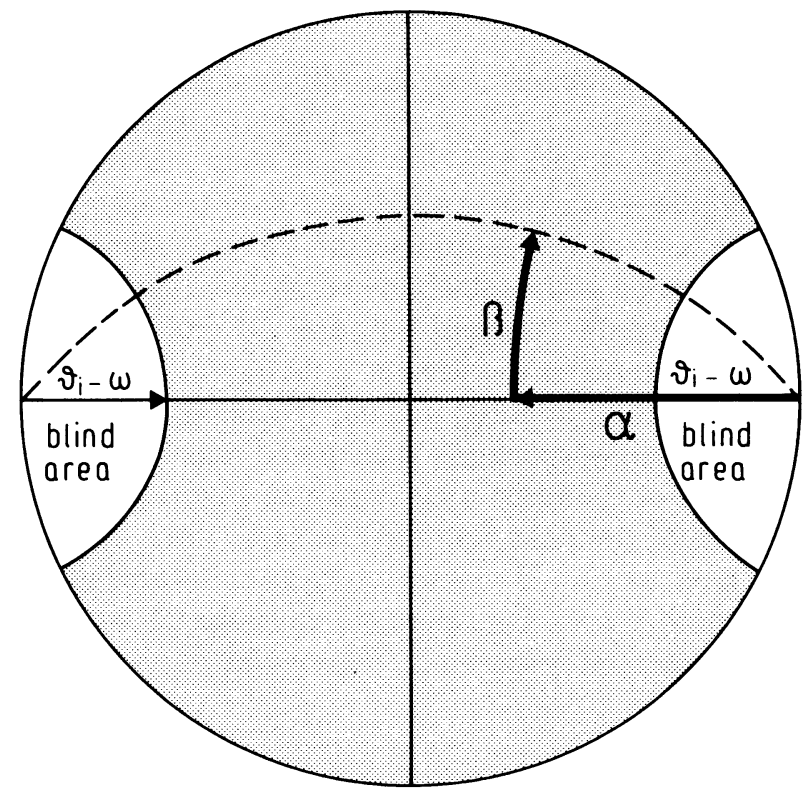

FIGURE 8 The blind area in the pole figure with $\vartheta_{i} \neq \omega$.

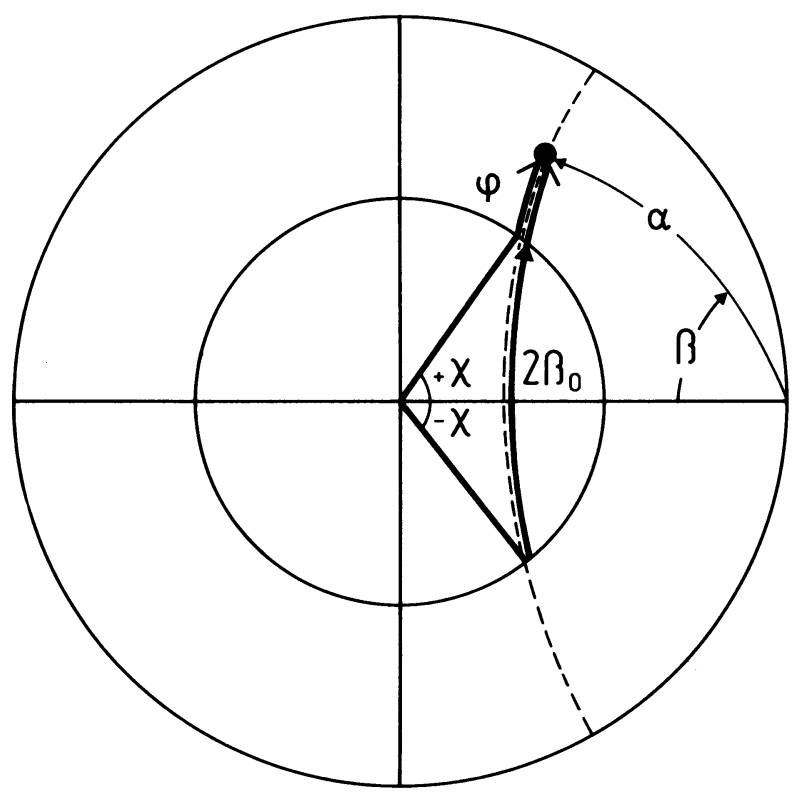

FIGURE 9 The same point of the pole figure can be obtained by two different sample orientations $(+\chi, \varphi)$ and $\left(-\chi, \varphi+2 \beta_{o}\right)$. 
of the pole figure can be obtained by two different settings of the sample

$$
\{\chi \varphi\}=\left\{-\chi, \varphi+2 \beta_{o}\right\}
$$

as is shown in Figure 9.

Because of the use of the multidetector all pole figures in the range $\vartheta_{1} \leqslant \vartheta_{i} \leqslant \vartheta_{2}$ are scanned at the same time i.e. with the same scanning pattern in $\chi$ and $\varphi$. Because of the relations between $\chi, \varphi$ and $\alpha, \beta$ this gives rise to different scanning patterns in the various pole figures. In order to minimize the measuring time with a required angular resolving power, the pole figure points $\alpha, \beta$ should be homogeneously distributed over the whole area of the pole figure. Because of the blind area shown in Figure 8 this is, in general, not possible for all of the pole figure at the same time. One can, however, choose an equal area scan in $\chi, \phi$ which leads to an equal area scan in $\alpha, \beta$ for the "central" pole figure $\vartheta_{i}=\omega$. One possible coverage of an equal area scan after Morris (pers. comm.) is shown in Figure 10.

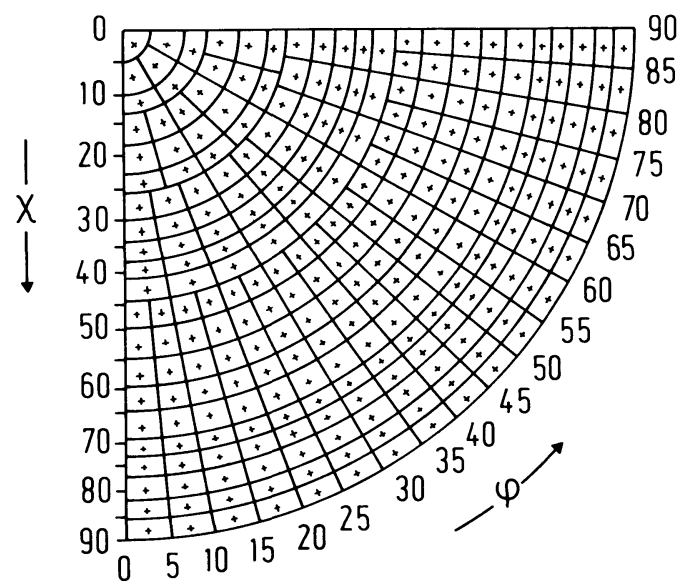

FIGURE 10 Equal area scan after Morris (pers. comm.)

In order to obtain points in the blind area (without removing the sample from its holder on the $\varphi$-shaft) it is necessary to vary also the angle $\omega$ in the range $\vartheta_{1} \leqslant \omega \leqslant \vartheta_{2}$. An equal area scan of the blind area of the $\vartheta_{2}$-pole figure is obtained for example by replacing $\chi$ with $\vartheta_{2}-\omega$ in the schedule of Figure $10, \varphi$ being the same as before and $\chi=0$. This is shown in Figure 11. When $\omega$ varies in the range $\vartheta_{2} \leqslant \omega \leqslant \vartheta_{m}$ then the whole blind area of the $\vartheta_{2}$-pole figure is obtained. At the same time points in the blind areas of all the other pole figures, the $\vartheta$-values of which are in the range $\vartheta_{2} \leqslant \vartheta \leqslant \vartheta_{m}$ are obtained, although not in an equal area mode. Another method to obtain points in the blind areas is to remove the sample from the $\varphi$-shaft and to 
rotate it through $90^{\circ}$ about the axis perpendicular to the $\varphi$-axis which is indicated by a dashed line in Figure 5. The blind area of the first sample setting (Figure 8) will then move into the centre of the pole (Figure 11) which can be measured with the above mentioned method.

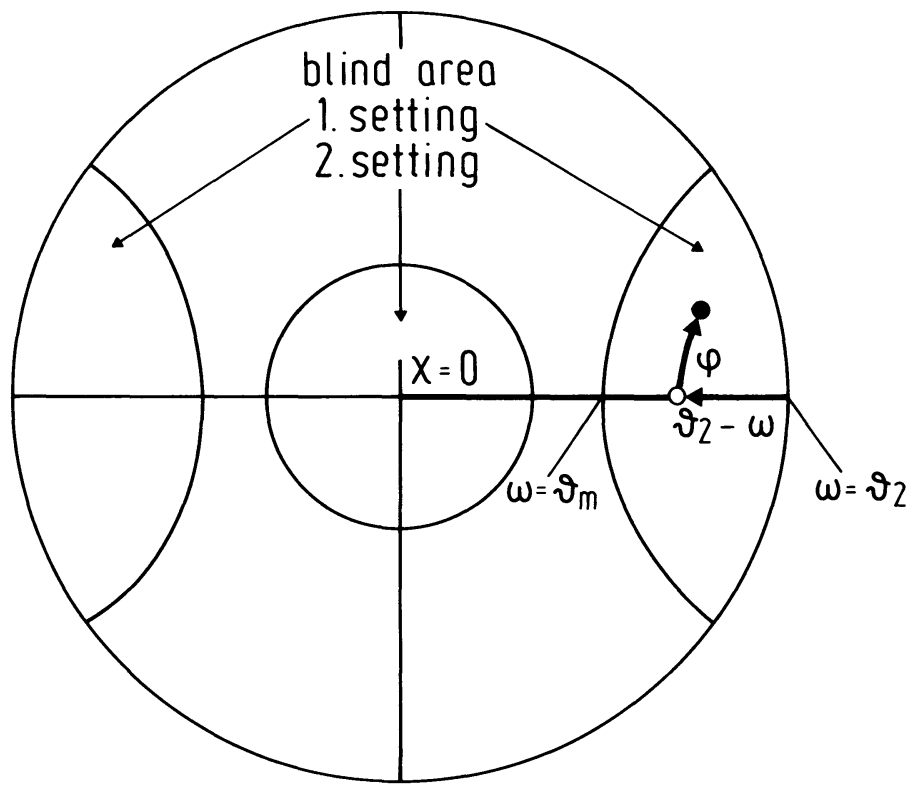

FIGURE 11 Points in the blind area can be obtained by an $\omega-\varphi$-scan in the first sample setting or by $\chi-\varphi$-scan in the second sample setting.

\section{THE ANGULAR RESOLVING POWER}

The angular resolving power in the pole figure is defined by the angular ranges $\Delta \alpha$ and $\Delta \beta$ through which a single crystal sample can be rotated without the reflected beam leaving the receiving slit. This corresponds to the angular range of orientations in an aggregate which reflect during a given measurement. We consider a rotation of the sample through the two angles $\omega$ and $\gamma$ (Figure 12). A possible rotation $\Delta \omega$ of the sample is determined by the divergency of the incident beam which, in turn, can be estimated from the half-maximum width in $2 \vartheta$ of the reflections. With the dimensions of the diffractometer shown in Figure 1 and a sample of about $8 \mathrm{~mm}$ in diameter this amounts to

$$
\Delta \omega \sim 0.25^{\circ}
$$


The range of rotation $\Delta \gamma$ can also be estimated from Figure 12. It is

$$
\Delta \gamma=\frac{h}{R \cdot \sin 2 \vartheta}
$$

where $h$ is the height of the detector receiving slit and $R$ the radius of the detector. With the actual values $h=100 \mathrm{~mm}, R=1522 \mathrm{~mm}$ one obtains

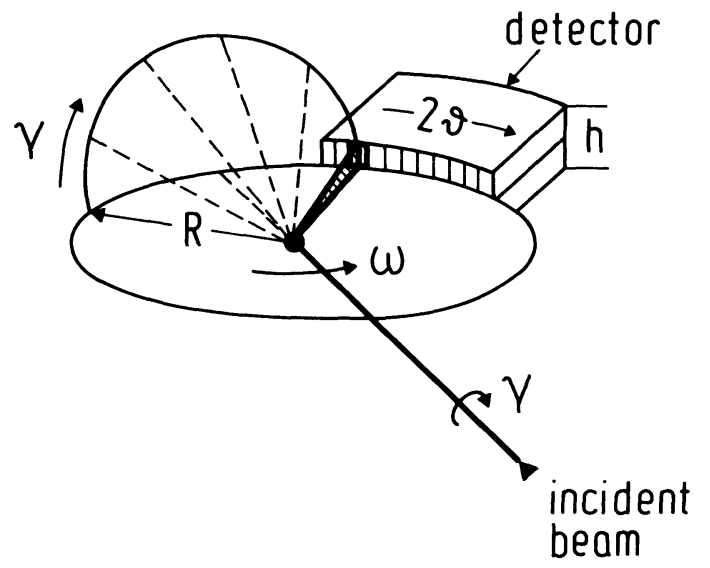

FIGURE 12 Determination of the resolving power in the pole figure.

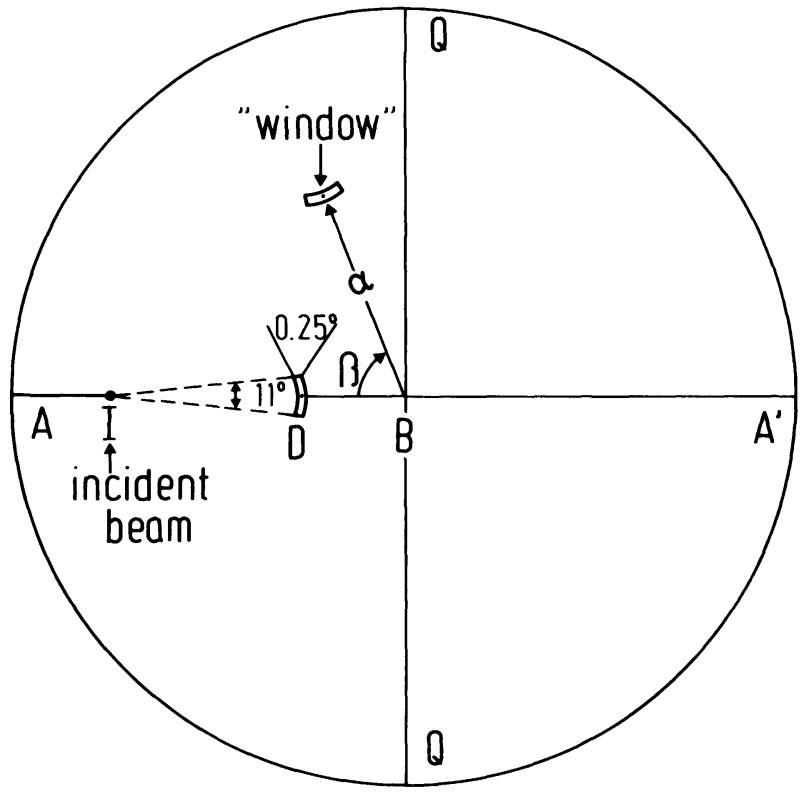

FIGURE 13 The "window" by which the pole figure is scanned. 


$$
\begin{array}{ll}
\Delta y=11^{\circ} & \left(2 \vartheta=20^{\circ}\right) \\
\Delta \gamma=5^{\circ} & \left(2 \vartheta=50^{\circ}\right)
\end{array}
$$

Hence, the height of the detector slit is the determining quantity for the resolving power of the method. The rotation $\Delta \gamma$ takes place about the incident beam $I$ (Figure 13) (compare also Figure 4 and Figure 7). The rotation $\Delta \omega$ is about the axis $Q-Q^{\prime}$. The shape of the window by which the pole figure is scanned is thus shown in Figure 13. It is rather small in one direction but large in the other one. For a sample with not too sharp a texture this resolving power is adequate but is insufficient for sharper textures. In this case higher $\vartheta$-angles must be used or the height of the detector slit must be reduced.

\section{POLE FIGURE SEPARATION}

As an example the neutron intensity as a function of $\vartheta$ for one sample position $\chi, \varphi$ is shown in Figure 14 for a sample of triclinic plagioclase feldspar with a large number of reflections. As the indexing of the peaks shows there is partial or complete overlapping of different diffraction peaks even in the low $\vartheta$-range. The problem then arises to separate the corresponding pole figures. It is a great advantage of the present method that it allows the use of two different and independent procedures for pole figure separation.

\section{Peak separation in $\vartheta$}

Part of the 9 scan of Figure 14 is shown in an enlarged scale in Figure 15. It is seen that there is a partial overlap of at least two diffraction lines (020) and (001). An overlap of this type can be separated by decomposing the curve into pure Gaussian peaks (which are the ideal peak shapes for neutron diffraction) using a least squares approximation

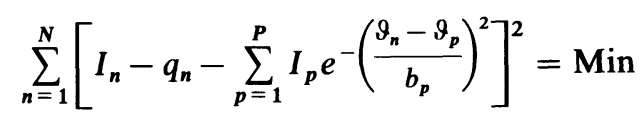

where $I_{n}$ is the measured value of the neutron intensity at the angle $\vartheta_{n}$ and $q_{n}$ is the background scattering. The quantities $\vartheta_{p}, I_{p}$ and $b_{p}$ are the position, peak height, and width respectively of the $p$-th Gaussian peak. These parameters can be calculated using an iterative least squares approximation programme (Wolfers, pers. comm.). The total reflected intensity $A_{p}$ corresponding to the peak $p$ is obtained by integrating the Gaussian function

$$
A_{p}=\int_{-\infty}^{+\infty} I_{p} e^{-\left(\frac{\vartheta-\vartheta_{p}}{b_{p}}\right)^{2}} d \vartheta=I_{p} b_{p} \sqrt{\pi}
$$


Of the three parameters $I_{p}, \vartheta_{p}$ and $b_{p}$ only $I_{p}$ depends on the pole figure angles $\alpha$ and $\beta$ whereas $\vartheta_{p}$ and $b_{p}$ must be assumed to be constant within one sample. It is thus sufficient to determine $\vartheta_{p}$ and $b_{p}$ only once for a sample. The minimum relation Eq. 9 is then simplified into a problem of linear approximation

$$
\sum_{n=1}^{N}\left[I_{n}-q_{n}-\sum_{p=1}^{P} I_{p} a_{n p}\right]^{2}=\operatorname{Min}
$$

where $a_{n p}$ are known coefficients

$$
a_{n p}=e^{-\left(\frac{\vartheta_{n}-\vartheta_{p}}{b_{p}}\right)^{2}}
$$

The values $q_{n}$ of background scattering can either be included in the minimization procedure or they can be interpolated from measured background values.

Eq. (12) must be solved for each sample position $\chi \varphi$. One thus obtains the integrated intensity of the peak $p$ corresponding to $\chi$ and $\varphi$ and hence to the pole figure angles $\alpha \beta$ (Eq. 1-3).

$$
A_{p}(\alpha \beta)=I_{p}(\chi \varphi) \cdot b_{p} \sqrt{\pi}
$$

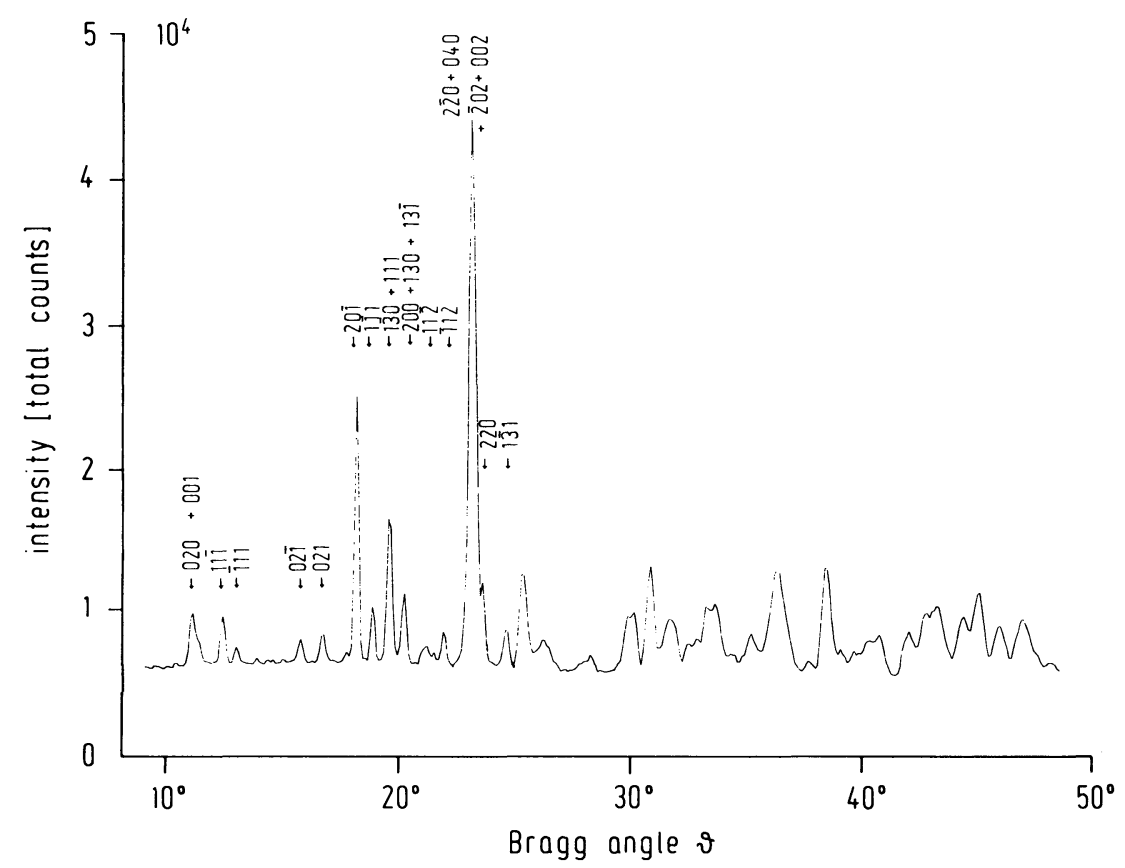

FIGURE 14 Neutron 9-profile of anorthosite mylonite from San Juan Bautista, Mexico. It is composed of Bytownite An 75. Some peaks are indexed. 


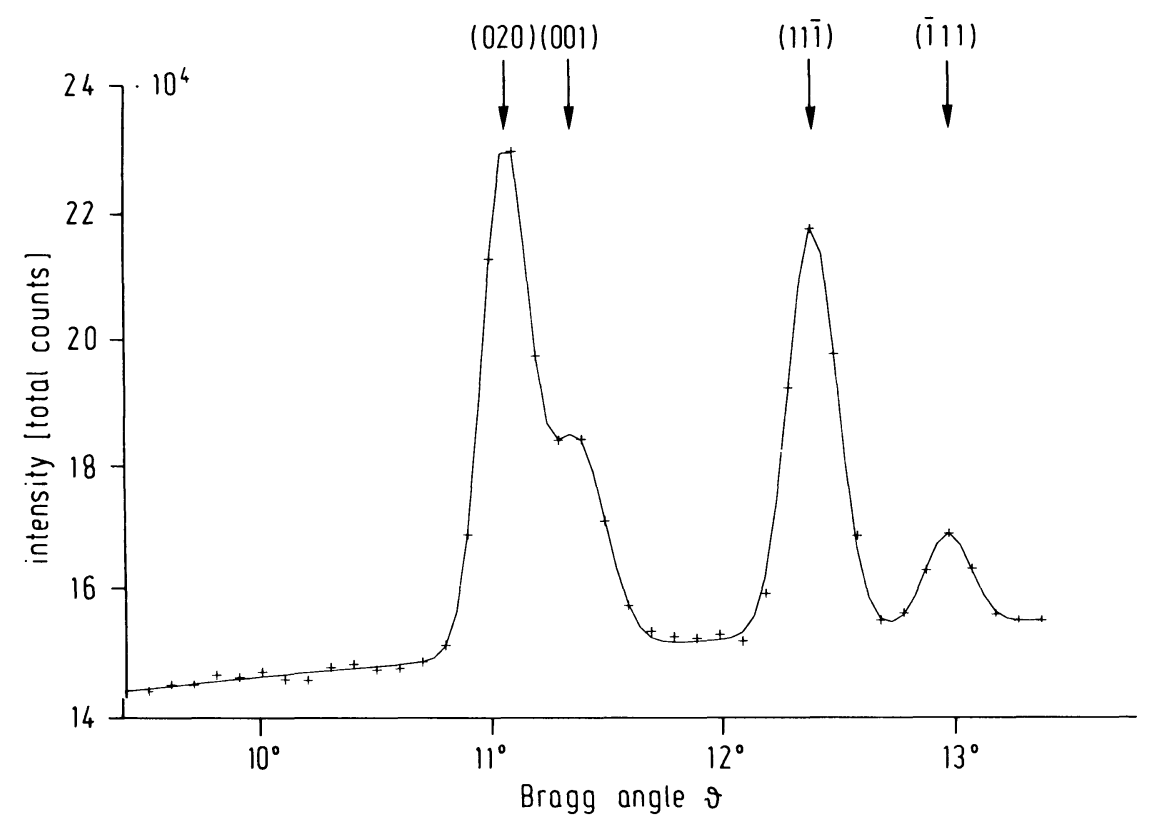

FIGURE 15 Enlarged part of the $\vartheta$-scan of Figure 14 showing partial overlap of two reflexions $(020)$ and $(001)$. The crosses represent experimental data measured in the individual cells. The solid line is the calculated least squares fit.

The pole density values are proportional to the integrated intensities

$$
P_{p}(\alpha \beta)=N_{p} A_{p}(\alpha \beta)
$$

The normalization factor $N_{p}$ can be obtained by integrating Eq. (14) over the whole pole sphere (Bunge, 1969) if

$$
\int P_{p}(\alpha \beta) \sin \alpha d \alpha d \beta=4 \pi
$$

Since, in this method, the pole figure is determined by integrated intensities rather than peak height measurements the counting statistics are better for a given time interval and the method is directly comparable to quantitative $\mathrm{X}$-ray single crystal diffractometry.

\section{Pole density separation in $\alpha \beta$}

Partial overlaps, as shown in Figure 15, can be separated by the method described in the section above. There are, however, other overlaps such as $(130)+(112)$ or $(130)+(13 \overline{1})+(200)$ in Figure 16 which are so close that it is not possible to separate them in the $\vartheta$-scan. Overlapped pole figures of 
this kind can be separated if one takes the complete distribution in $\alpha \beta$ into account. The principle of this separation is shown in Figure 17. Although the two pole figures $J_{1}(\alpha \beta)$ and $J_{2}(\alpha \beta)$ are so close together that they cannot be separated in $\vartheta$ they are quite different in $\alpha$ and $\beta$. The two distributions $J_{1}(\alpha \beta)$ and $J_{2}(\alpha \beta)$ must be consistent with the distribution in all the other pole figures. Because of the crystallographic relationships between them the various pole figures of a texture are not independent of each other. Rather, they are interrelated in such a way that they are different two-dimensional projections of the same three-dimensional orientation distribution function. This method of pole figure separation has been worked out in terms of the series expansion method of texture analysis (Bunge, 1969). The combination of the two separating methods thus allows the extraction of the maximum information from the experimental data.

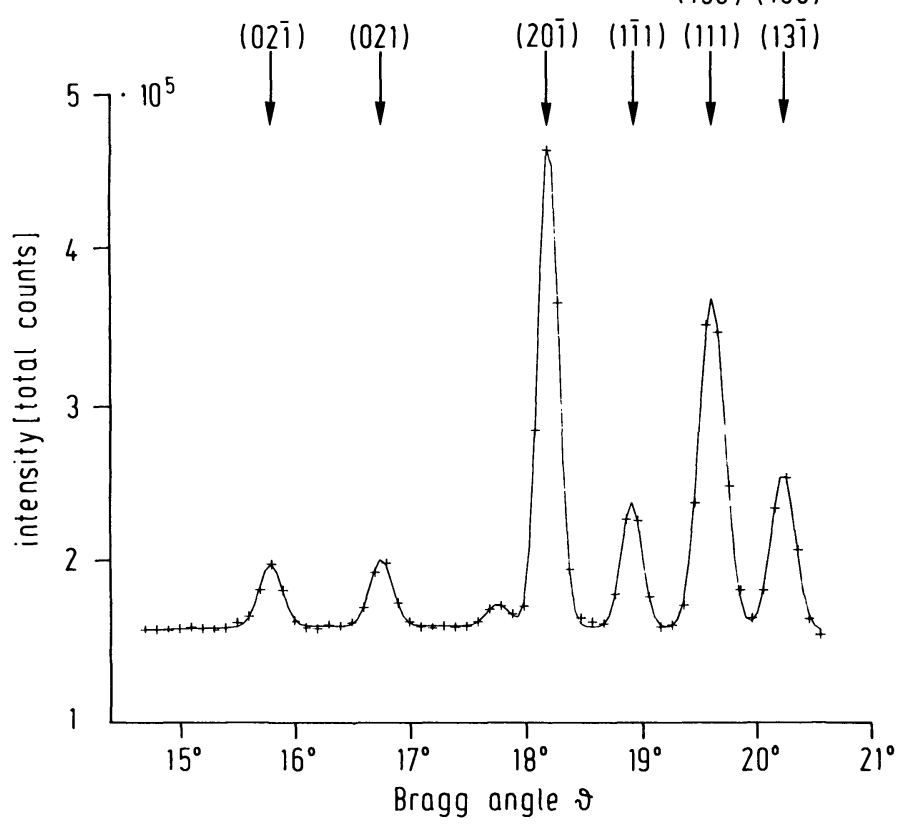

FIGURE 16 Enlarged part of the 9 -scan of Figure 14 showing total overlaps of two reflexions $(130)+(111)$ and $(130)+(13 i)+(200)$. The crosses are experimental values. The solid line is the calculated least squares fit. 


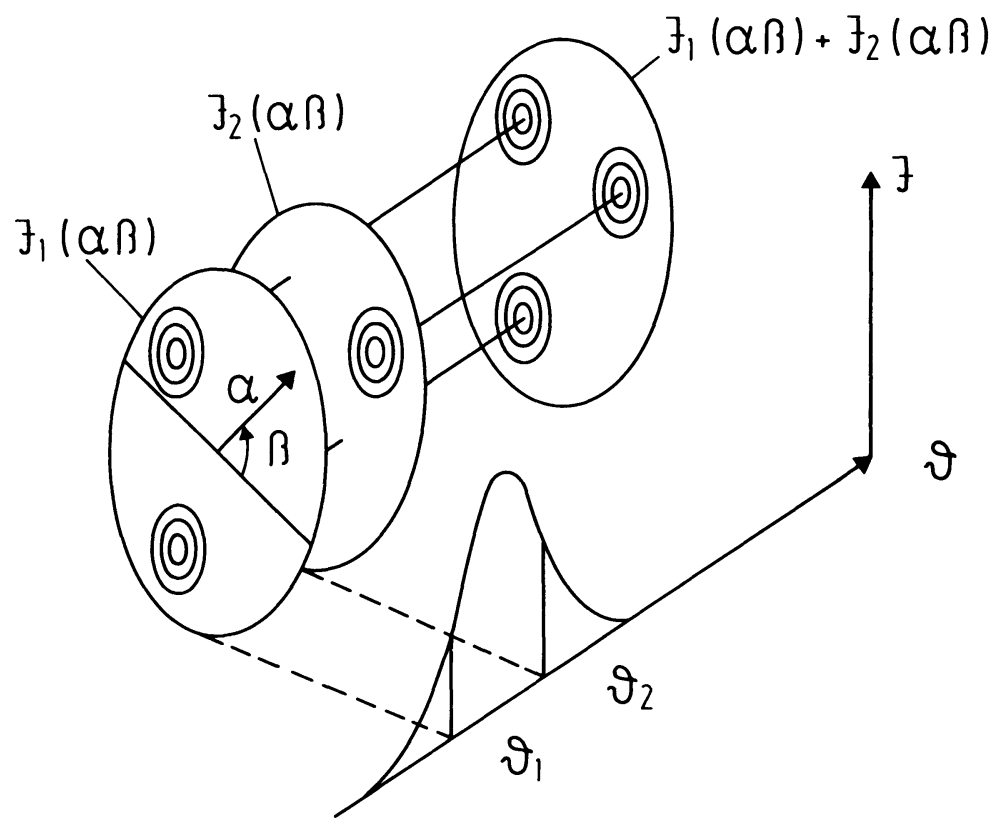

FIGURE 17 Superposition of two pole figures $J_{1}(\alpha \beta)$ and $J_{2}(\alpha \beta)$ with neighbouring $2 \vartheta$-values.

\section{CONCLUSION}

The method outlined above takes into account the maximum information which can be obtained by a diffraction experiment from a textured polycrystalline sample because complete spectra are measured over the Bragg angle $\vartheta$. The use of a position sensitive detector allows the spectra to be measured in a reasonable time. And finally, neutron diffraction, because of its lower absorption coefficient, allows one to use a sufficiently large sample compared with grain size. The method is thus very well suited for the determination of textures in materials having complex diffraction spectra i.e. materials with low symmetries and large unit cell parameters as they frequently occur in geology.

We are in the process of applying the above mentioned method to a specimen of anorthosite mylonite with triclinic crystal and triclinic specimen symmetry.

\section{Acknowledgements}

The authors want to thank the Institut Laue-Langevin for providing neutron diffraction facilities. The project was partly sponsored by the Deutsche Forschungsgemeinschaft. One of the 
authors, H. R. Wenk, wants to express his gratitude to Humboldt foundation. Figure 1 is reproduced from the ILL neutron beam facilities, edited by the office of the scientific secretary (1981).

\section{References}

Allemand, R., Bourdel, J., Roudaut, F., Convert, P., Ibel, K., Jacobe, J., Cotton, J. P. and Faroux, B. Nucl. Instrum. Methods 126, 29 (1975).

Bunge, H. J. Mathematische Methoden der Texturanalyse. Akademie Verlag, Berlin (1969).

Bunge, H. J. Tectonophysics 78, 1 (1981).

Convert, P. Thesis - Grenoble (1975).

Gaglioti, G., Pauletti, A. and Ricci, F. P. Nucl. Instrum. Methods 3, 223 (1958).

Loopstra, B. O. Nucl. Instrum. Methods 44, 181 (1966).

Wassermann, G. and Grewen, J. Texturen metallischer Werkstoffe. Springer-Verlag Berlin, Göttingen Heidelberg (1962). 